To be published in

IEEE Trans. Nuc1. Sci.

Presented at IEEE Nuclear Science

Symposium, 19-21 October 1977,

Sheraton Palace Hotel, San Francisco

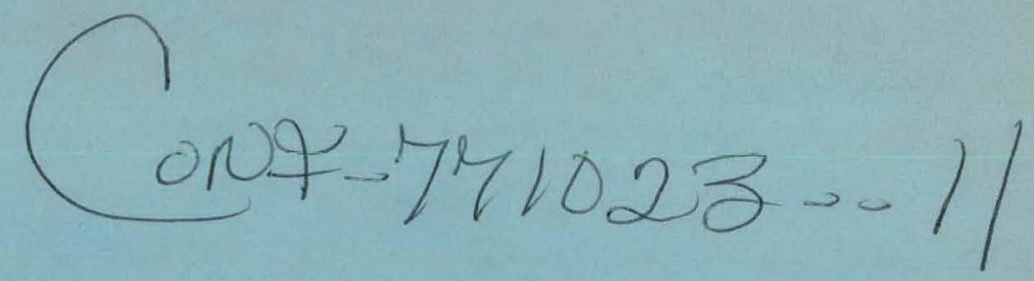

MASTER

\begin{abstract}
A MAIN PROGRAM AND OVERLAY MANAGER SUBSYSTEM
WITHIN A DISTRIBUTED FUNCTION LABORATORY COMPUTER SYSTEM*
\end{abstract}

F. W. Stubblefield

Brookhaven National Laboratory

Upton, New York 11973

October 1977

*This research was supported by the U. S. Department of Energy:

Contract No. EY-76-C-02-0016. 


\section{DISCLAIMER}

This report was prepared as an account of work sponsored by an agency of the United States Government. Neither the United States Government nor any agency Thereof, nor any of their employees, makes any warranty, express or implied, or assumes any legal liability or responsibility for the accuracy, completeness, or usefulness of any information, apparatus, product, or process disclosed, or represents that its use would not infringe privately owned rights. Reference herein to any specific commercial product, process, or service by trade name, trademark, manufacturer, or otherwise does not necessarily constitute or imply its endorsement, recommendation, or favoring by the United States Government or any agency thereof. The views and opinions of authors expressed herein do not necessarily state or reflect those of the United States Government or any agency thereof. 


\section{DISCLAIMER}

Portions of this document may be illegible in electronic image products. Images are produced from the best available original document. 
To be published in

IEEE Trans. Nucl. Sci.

Presented at IEEE Nuclear Science

Symposium, 19-21 October 1977,

Sheraton Palace Hotel, San Francisco

\title{
A MAIN PROGRAM AND OVERLAY MANAGER SUBSYSTEM WITHIN A DISTRIBUTED FUNCTION LABORATORY COMPUTER SYSTEM*
}

\author{
F. W. Stubblefield \\ Brookhaven National Laboratory \\ Upton, New York 11973
}

October 1977

This report was prepared as an account of work sponsored by the United States Government. Neither the United States nor the United States Department of Energy, not any of their employees, nor any of their contractors, subcontractors, or their employets, makes lia warranty, cxpress or implied, or assumes any legal harihy or responsibility for the accuracy, comple teness or usefuiness of any information, apparatus, produrft or pivess disclosed, or represents that its use would not infringe privately owned rights.

* This research was supported by the U. S. Department of Energy: Contract No. EY-76-C-02-0016. 
A MAIN PROGRAM AND OVERIAY MANAGER SUBSYSTEM

WITHIN A DISTRIBUTED FUNCTION LABORATORY COMPUTER SYSTEM*

\section{F : W. Stubblefield}

Brookhaven National Laboratory

Upton, New York 11973

\section{Abstract}

A key property of modern systems to control and acquire data from a collection of laboratory experiments is that the computer programs to effect this control and data acquisition are written in a highlevel language, e.g., FORTRAN. The large amount of core memory required for execution of programs compiled from the FORTRAN language poses the most significant problem in the design of systems to support this language. A comnon method of solving this problem involves dividing the execution code into a main program and a set of associated overlays. A subsystem for developing and executing FORTRAN programs having such a main program-overlay structure can be implemented in many ways. The details of an implementation within a functionally distributed laboratory computer system are given. Emphasis is placed on the description of an auxiliary fast-access disk used as an intermedlate storage device for read-only access information.

\section{Introduction}

A key property of modern computer systems for experiment control and data acquisition is that the computer programs to effect this control and data acquisition are written in a high-level language. Since many scientists have had at least a minimal amount of experience with FORTRAN, this language is an excellent choice for the implementation of such highlevel routines. An additional advantage of FORTRAN is that it is a compiled language. The compilation process serves as a rapid pre-execution filter for syntax errors in the program language statements.

The compiled-language property of FORTRAN becomes even more important in the particular case of computer systems for experiment control and data acquisition. During a major portion of the time that such a system is In operation, the system processors as well as the experiment electronics must be utilized to service on-going experiments; only a small fraction of the time is available for testing new computer programs and modifications to existent ones. Hence, provision must be made for eliminating as many errors as possible from these programs prior to actual execution.

The most serious liability of a high-level language is the relatively large amount of core memory space required to implement a particular function as compared to the space required to implement the same function using a lower level language. It is not unusual for the total space requirements of high-level language programs for experiment control and data acquisition to reach $10^{5}-10^{6}$ core memory words, a total which exceeds the space avallable in most computer processor-main memory configurations. (Here, a main memory word consists of 16 bits.)
The problem of the exceptionally large core memory requirements of high-level languages can be converted into lesser problems by sharing the memory in time among various portions of the program. The algorithm for deciding which portion of the program is to reside in memory at any given time is coded either explicitly or implicitly into the program itself. Since this program is written by the system user (experimenter), this solution places an additional burden on this person. In particular, each user must have a detailed understanding of the following:

(1) the program structure tmposed by the program partitioning method. This includes the definition of the logical program units which may share the available core memory and the hierarchical relationship among the logical units;

(2) the method for communicating information between the logical program units;

(3) the physical storage space restrictions imposed by the avallable memory on both the individual logical units and various combinations of these units;

(4) the method for programing the sharing algorithm in the high-level language.

Thus, one of the decisions to be made before implementing a high-level language for experiment control and data acquisition is whether or not these additional burdens on the user can be tolerated in order to obtain the many advantages which these languages afford. In the present case, it has been determined that the additional problems do not become unmanageable if the program partitioning method adopted is the simplest possible one.

\section{High-Level Language Partitioning Method}

As mentloned above, the high-level language selected for implementation at the present system is FORTRAN. A standard method exists for partitioning FORTRAN program code. A part of the total code, the logical unit termed the main program, is made permanently resident in core memory. Other parts of the code, the logical units termed overlays, occupy at different times a portion of the memory separate from that occupied by the main program. Also, a set of contiguous areas in the space occupied by the main program logical unit, the set of common areas, can be defined and utilized for communicating information among the logical program units. This structure is the only part of the FORTRAN memory partitioning

* This research was supported by the U. S. Department of Energy: Contract No. EY-76-C-02-0016. 
scheme which is standardized. In particular, the requests to load the overlays to main memory can be made explicit or implicit and the structure (layout) of the overlays in memory can attain various degrees of complexity, the most complex being an autoloaded overlay system with a tree-structured call and layout scheme. Similiar complexities can be introduced into the definition and layout of the common areas. In the present system such schemes are considered to be much too complex for an average user to understand easily. The properties of the present FORTRAN overlay system are the following:

(1) the main program has a fixed maximum length of 8192 16-bit words of memory;

(2) all common areas for main programoverlay communication have their structure defined and their required memory space allocated in the main program;

(3) each overlay has a fixed maximum length of 8192 16-bit words of memory;

(4) only one overlay may be present in memory at any time;

(5) all requests to load and execute overlay code are generated by explicit program statements in the main program.

\section{System Architecture}

The computer system upon which the FORTRAN programming system has been implemented is designed according to a functionally distributed architecture. The principles upon which this architecture is based have been given elsewhere. 1 An overvlew of the design and implementation of this computer system, the Reactor Experiment Control Facilfty at Brookhaven National Laboratory, has also been published. ${ }^{2}$ The first step in designing a computer system according to a functionally distributed architecture is to identify and state the system function. The next step is to partition this function into subfunctions which reside at a lower level of functional complextty. The design work is continued by iterating the partitioning operation until a set of functions is located which can be confined to a node of the system, where a node contalns all of the hardware and software required to implement each function in the set, and, furthermore, this hardware and software is not used to implement any function not within this set. The advantages which accrue to a computer system consisting of a set of nodes which perform functions assigned to them in this manner have been fully listed elsewhere. 1

The system function of the Reactor Experiment Control Facility is to "control and monitor and acquire data from a set of laboratory experiments". The first partitioning operation performed on the system function has a major Impact on the feasibility of implementing a high-level language programing subsystem. In the present case, the system function is partitioned into the two functions:

(1) develop programs for experiment control and data acquisition, and

(2) perform operations required to control and acquire data from a set of laboratory experiments.
The program development function is very nearly a node level function as it stands, i.e., it is almost possible to completely confine it to a system node. However, one additional partitioning operation must take place. The function ( 1 ), above, is partitioned into the two subfunctions "generate programs for experiment control and data acquisition" and "file (store) executable coples of programs for experiment control and data acquisition". The first of these subfunctions is confined to a node of the system and this node is labelled the program development node. The second subfunction can also be confined to a system node, the shared service or common node. In this case, however, additional subfunctions are also performed by the node.

The function (2), above, is not so easily confined to a single system node, and, in fact, many advantages 1,2 to the system as a whole can be realized if this function is further partitioned before confinement. This function is next partitioned into the function "provide a set of shared services required to control and acquire data from a set of laboratory experiments" and a set of $n$ functions "execute program to control and acquire data from experiment number $x "$ ", where $n$ is the total number of laboratory experiments. Each of these $n$ functions is confined to a private execution or appilication node.

The function of providing a set of shared services must be partitloned once more. The three resulting functions are the following:

(1) request services to initialize and manipulate the set of shared services;

(2) supply services to initlalize and manipulate the set of shared services;

(3) supply a set of shared services required to control and acquire data from a set of laboratory experiments.

The first of these functions is confined to a system control and manipulation node. The second two are combined with the function "file executable copies of programs for experiment control and data acquisition", mentioned above, and all are assigned to the common or shared service node, also mentioned above.

Once the system structure has been reviewed, a discussion of the FORTRAN programming subsystem reduces to a description of its implementation at the varfous nodes of the system. Since the point of most interaction of all the laboratory experiments serviced by the system is the comon node, implementation of the subsystem at this node will be emphasized.

\section{Program Development Node}

Al1 computer programs for experiment control and data acquisition are constructed at the program development node. Hardware and software at this node are used to generate high-level language source code and reduce this source code to core-image (executable) form. The node implementation elements are discussed below.

\section{Hardware Implementation Elements}

Hardware elements present at the program development node include the following: 
(1) a computer processor ${ }^{3}$ and 28,672 words (16-bit) of associated core memory;

(2) a moving head disk controller 4 and dual drive for one 1.2 megaword removable platter and one 1.2 megaword non-removable platter;

(3) a small capacity random-access magnetic tape controller $r^{5}$ and dual tape drive;

(4) a punched card reader 6 capable of reading at a rate of 300 cards per minute;

(5) an electrostatic line printer ${ }^{7}$ capable of printing at a rate of 500 lines/minute;

(6) a keyboard terminal device ${ }^{8}$ capable of transferring characters at a rate of 30 characters/second.

\section{Software Imp lementation Elements}

Software elements utilized at the program development node include the following:

(I) a single-user disk operating system; 9

(2) a character string oriented text

(3) a FORTRAN language comp1ler;

(4) a macro language assembler;

(5) a library processor for maintaining library files of relocatable modules of object code;

(6) a linker routine for resolving external references within object modules and assigning the object modules to places in logical address space;

(7) a file transfer routine for transferring files between peripheral devices;

(8) a l1brary 11 of assembly language level subroutines to support execution of FORTRAN compiled code by the operating system;

(9) a library of assembly language level subroutines to support FORTRAN-standard subroutine calls which reference special purpose experiment electronics.

\section{Program Development Node Operation}

Three fundamentally different operations are carried out at the program development node: generation and modification (editing) of source code, compilation of source code to relocatable binary (object) modules, and linking of object modules to logical (and physical) address space. The program development node is always used in single-user mode; only one user can perform che operativins discussed below at any one time. While utlization of a processor in the singleuser mode may not be justified in all instances, in the particular case of a system for experiment control and data acquisition, it has been found to be satisfactory

Source Code Editing. The medium for permanent storage of user source code is DECtape, 12 the small capacity reels of randomly accessed magnetic tape mentioned above. Source code is usually generated by typing characters at the keyboard terminal and having the characters stored in a file on the node disk. This code generation process is controlled by the editor program. Source modification is accomplished In a simllar manner; the source file on DECtape is copied to disk, the editor program is used to modify the disk file, and the disk file is copied back to DECtape. Some users, however, have found an alternative method of source code maintenance to be more convenient. All source code generation and modification is done on punched cards (IBM 026 punch). The latest version of the code is transferred from the card reader to a DECtape file prior to initiating the compilation and linking operations.

Source code Compllation. In order to compile a main Program or overlay, the user has only to position two DECtapes on their respective drives and invoke a batchstream of job control commands by typing a sixcharacter file name. A DECtape containing the source code to be compiled must be positioned on one drive, with the object code library Dectape placed on the other. Any other information required during the compliation process is solicited from the user (via prompting questions at the keyboard terminal) by programs called into execution by the batchstream commands. Thus the compilation process is completely automatic. The following items of information are produced by the batchstream:

(1) a listing of the FORTRAN code for the main program or overlay which indicates any syntax errors located by the compiler;

(2) a directory of the contents of the object code DECtape. This directory shows which overlays have been compiled and have object code present.

Object Code Linking. The process of linking a FORTRAN maln program or overlay to its external references and assigning its executable code to a place in main memory is also initlated by invoking a batch: stream. The FORTRAN main program must always be linked first, 1.e., before any overlays are linked. The output information generated by this batchstream consists of a list of global 13 symbols (a symbol table) which give the locations of common block variables, the entry points to subroutines contained within the main program, and the location of the main program entry point in main memory. This information is also printed on the line printer in the form of a load map.

The linking process for an overlay is similar except that the symbol table serves as part of the input to the process. Entries within the symbol table are used to satisfy extemal references made from within the overlay to the main program common area and to subroutines located in the main program memory space. One symbol table entry is also used to determine the placement of the overlay code in physical main memory. A load map giving the entry points of the overlay code 
and all subroutines linked into the overlay main memory area is also printed on the line printer.

The last two operations performed by the LINR MAIN PROGRAM and LINK OVERLAY batchstreams are to reduce the relocated code to core-image executable form and to transfer the core image to the common node by requesting the PUT MAIN PROGRAM or PUT OVERLAY transaction function.

\section{Control and Manipulation Node}

Operations which initialize and manipulate application-node-global information maintained by the FORTRAN programming subsystem are initiated at the system control and manipulation node by the system operator. (Application-node-global information is control information which can have an effect on more than one application node if it is changed.) In general it can be shown 14,15 that the software and hardware elements required to implement this node are a subset of those required to implement the program development node. While the control and manipulation node is very obviously a logically separate node and very strong reasons can be advanced for its Implementation as a physically separate node, economic reasons usually make such a separate implementation unfeasible. Such is the case in the present system. The control and manipulation functions and the program development functions are performed by the same physical node. Thus the software and hardware elements listed above are also present at the control and manipulation node. Only one subsystem operation is initiated at the control and manipulation node. A batchstream to request the INITIALIZE function is maintained on DECtape and executed by the system operator whenever the entire FORTRAN programing subsystem is to be cleared of all information and restarted. Typically, the time period between INITIALIZE function requests is 1-2 years.

\section{Application Nodes}

Main programs and overlays generated at the program development node are executed at the application nodes. Hardware present at an application node includes as a minimum a Digital Equipment Corporation PDP-11/40 processor and 24,576 words of 16 -bit main memory and a Texas Instruments 700 keyboard terminal. Each application node also includes special devices (experiment electronics) to control the detectors present at the experiment and to store the data produced by the detectors into memory buffers.

Application node software includes an in-house developed operating system to support FORTRAN program execution. This operating system is completely coreresident and resides within 8,192 words of main core memory. The operating system is essentially the Digital Equipment Corporation Disk Operating System, Version 9, with all support routines removed except those required for the terminal device. Routines for servicing the special experiment dependent devices reside in the main program and overlay areas as assembly language level routines accessed via FORTRANstandard subroutine calls.

At the beginning of an experiment, one routine within the application node operating system solicits from the user the six-character name of the main program to be used to control and acquire data from the experiment by printing a question at the keyboard terminal device. After the user responds, this routine utilizes the name as a parameter of a GET MAIN
PROGRAM transaction submitted to the common node. At the successful conclusion of this transaction request, the requested main program resides in application node main memory and is automatically entered from the operating system.

As mentioned above, the requests to load overlays to main memory and begin their execution are explicitly coded into the FORTRAN main program. These requests take the form of a call to a system standard subroutine. The main parameter of this subroutine call is the overlay number. A call to this subroutine has the result that the GET OVERLAY transaction function is requested of the common node, the overlay is transferred to the application node and loaded into main memory and its execution begun. The flow diagram of the execution sequence from FORTRAN main program through routines which drive the inter-node communication link has been given elsewhere. 15 It should be noted that GET MAIN PROGRAM and GET OVERIAY are the only transaction functions belonging to the present subsystem which can be requested by an application node.

\section{Common Node}

The function of the common node is to supply a set of shared services to the private nodes, 1 .e., the control/manipulation node, the program development node, and the application nodes. The hardware present at this node and the node operating system and set of task level subsystems have been described in much detail elsewhere. 14-18 Only a very brief review will be given here.

The comon node has been implemented as a transaction processor. Requests for service directed to this node and the subsequent response to these requests take the form of a transaction, where a transaction consists of two mandatory and one optional transmissions over the communication link between the common node and the private node requesting the service. Transactions entering the common node have some very definite properties 18 of which two should be stated here:

(1) the elapsed time required for complete processing of any transaction is $10^{-2}-10^{0}$ seconds;

(2) all transactions have approximately equal weight with respect to their response time requi rements.

Each transaction is processed by two task sequences. The first sequence accesses information in the REQUEST transaction parameter block and assembles an ACKNOWLEDGE transaction parameter block for transmission back to the requesting private node. If the optional transaction DATABLOCK transmission is required, the second task sequence finishes the transaction processing after this transmission has taken place.

While processing a transaction, each task in these sequences may access common node resources. 16,17 Statically allocated resources consist of modules of subroutines, read-only access tables, and modification access tables which are associated with the various task level subsystems. Logical resources are structured flags which represent access, both read-only and modification, to various information structures within the common node. Physical resources are contiguous blocks of main memory which can be used as control blocks for executing various input/output operations or as buffers for these operations. 
The overall scheme for processing transactions in the common node is the following:

All resources, both $\log 1$ cal and physical, required to completely process a transaction are claimed prior to the start of transaction processing.

In this way the lockout problem due to conflicting resource requirements of two simultaneously processed transactions is avoided.

The task level subsystems which process the transactions and an operating system 19 to support task execution comprise the software present at the common node. The hardware consists of a Digital Equipment Corporation PDP-11/45 computer with three modes of memory management and a set of high-quality shared peripheral devices. Among these devices is a movinghead disk controller and a single 20 megaword disk drive.

\section{Main Program and Overlay Manager Subsystem}

The task level subsystem which manages the executable core images of FORTRAN programs at the common node is the main program and overlay manager subsystem. The tasks in this subsystem and the transaction functions which they perform are listed in Table I.

Images of main programs and overlays are maintained in a data partition of the 20 megaword disk mentioned above. (In what follows, this disk will be referred to as the "slow" disk.) This partition contains 6,291,456 16-bit words, so that a maximum of 1,536 main programs or overlays can be stored. The directory and allocation map required to manage this data partition are maintained in a control partition of the same disk. This control partition contalns 135,168 words ( 33 blocks of 4,096 words each), a space which is sufficient for the management of $256 \mathrm{maln}$ program entries. The arrangement of information contained in a main program directory entry is shown in Fig. 1. The allocation map contains an 8-bit byte for every 4,096-word allocation unft available in the data partition. While it is true that every main program and overlay has a maximum possible length of 8,192 words, a sufficient number of overlays shorter than or equal to 4,096 words in length exists to warrant allocating the data partition in 4,096-word blocks.

Most transactions require the following resources for their complete processing:

(1) a logical resource which represents access to the main program and overlay manager subsystem directory, allocation map, and data partition. As mentioned above, this logical resource can be requested for elther read-only or modification access to these areas;

(2) a physical resource of length 4,096 words. This resource is used as an input/output buffer for transfers of the subsystem directory from/to the slow disk;

(3) a physical resource 8,192 words in length. This resource ts used as a transaction datablock and contains the main program or overlay core lmage being transferred to or from the slow disk or to or from a private node via a communication ifnk.

In general, this management scheme has been found to be satisfactory under actual system operation. Most of che subsystem functions require $\sim 500$ mflliseconds for their performance. This response time is satisfactory because most of the functions are requested only every few hours. This is true of all functions requested by the control/manipulation and program development nodes. It is also true of the GET MAIN PROGRAM function requested by the application nodes. Typlcally a main program is requested at the start of an experimental run and may remain undisturbed in the main memory of an application node for several weeks. However, one function which must be provided by the main program and overlay manager subsystem is requested much more often. Typically an application node may request the GET OVERLAY function almost continuously for short perlods of time $\left(10^{0}-10^{1}\right.$ seconds). This is especially true when an experimental run is being set up. Users like to program the various logically separate experiment initialization operations as separate overlays and call them one-after-another in sequential fashion during experiment start-up. While the 500 millisecond response time is satisfactory, it is apparent that any fmprovement in this time which could be made without radically changing the system would be well recelved by the users.

An improvement is possible both because of the strict division maintained in the common node software between read-only and modification access information and because of the functional definition of the common node as a transaction processor. Each transaction entering the common node can be classified according to whether or not it changes information maintained within the node. This means that temporary information can be maintained within the node and utilized by tasks which require only read access to common node information in order to perform their transaction processing. Upon receipt of a transaction which modifles information managed by the node, all temporary information can be cleared, the master copy of the cemporary information updated, and the process of reconstructing the temporary copy begun anew. The reconstruction process continues and temporary information accumulates (or remains undisturbed) until the next transaction requiring modification access to the information is received.

\section{Extension of the Subsystem}

As mentioned above, the point at which the main program and overlay manager subsystem can be fimpoved is the GET OVERLAY function. The total time required to perform this function is $\sim 500$ milliseconds. The times which contribute to this total are listed in Table II. The disk used to Implement the subsystem has an average access time (cylinder positioning time plus half rotation time) of 41.5 milliseconds and a transfer rate of $7.5 \mathrm{\mu sec}$ per $16-b i t$ word. Thus 94 milliseconds are required to read the subsystem master program and program directories from the disk. If it is assumed that the average length of an overlay is 4,096 words, an additional 72 milliseconds are required to read the overlay 1tself. Also, in the worst case (maximum length overlay), 197 milliseconds would be required for these two transfers. While a master copy of the subsystem information must always be maintalned, it is possible to reduce the disk access and transfer time by keeping a temporary copy of this information in main memory or 
on a faster disk.

In the extended subsystem coples of the most frequently used overlays are stored on a Digital Development Corporation fixed-head disk. 20 The access and transfer properties of this disk are an average access time (half rotation time only) of 8.7 milliseconds and a transfer rate of $4.2 \mathrm{\mu sec}$ per word. Thus the average 4,096 word overlay can be transferred to main memory in 17.2 milliseconds. A much condensed version of the subsystem directory is maintained in main memory. When a frequently used overlay is requested for the second time with no intervening modification access transaction, its core image can be placed in the transaction datablock in an average time of 26 milliseconds. Thus the total transaction processing time is reduced by $\sim 30$ percent.

The algorithm for diminishing the amount of temporary information is simple. Whenever a transaction is processed which must modify any of the subsystem information, the directory in core memory is cleared. This operation also implicitly removes all overlay images from the fast disk. As subsequent requests for overlays are received, a copy of each overlay is written to the fast disk and its main program name and overlay number are entered into the temporary main memory directory. When the fast disk partition is full, the least frequently used overlay is removed and replaced with a copy of the currently requested overlay. The overlay frequency-of-use is determined by maintaining a linked chain of pointers to the overlays (in the memory resident directory) and keeping the currently requested overlay at the head of the chain.

\section{Logical Resource Manipulation}

As mentioned above, master copies of the overlays are maintained on the slow disk. In general, several transactions processed by the main program and overlay manager subsystem could be in progress at any one time. Thus it would be possible, for example, for transaction A to read a copy of the subsystem directory, allocate space for a new overlay, update its copy of the a1location byte map, and yield the processor in order to write the directory back to the slow disk. Before this write operation has been completed, however, transaction $B$ could obtain a copy of the original directory, allocate space for a new overlay, and itself yield the processor in order to write its copy of the directory back to the slow disk. Eventually the directory copy due to transaction $B$ would be written over the copy due to transaction $A$ and the overlay added by transaction $A$ would disappear. In order to prevent such overlapping update operations by different transactions, the logical resource construct is utilized. The main program and overlay manager subsystem logical resource is a member of the group of transaction processing resources claimed by the initial task in all REQUEST phase sequences which process transactions directed toward this subsystem. Modification access to this logical resource is relinquished only when all operations which modify the information which this resource represents have been completed. Transactions which access the subsystem information in readonly fashion claim the resource for read-only access before proceeding. The algorithm for granting access to the resource dictates that multiple transactions may claim read-only access at one time but all such transactions must have relinquished access to the resource before it can be assigned to a transaction requiring modification access. Also, only one transaction at a time may hold modification access. In this manner, every transaction is guaranteed that the first obtained copy of the information to which the logical resource represents access w1ll remain current throughout the complete processing of the transaction.

In order to extend the subsystem, an additional logical resource must be defined. This logical resource represents access to the temporary d1 rectory maintained in main memory. Every GET OVERLAY transaction must include this logical resource with modification access in its initial group of requested resources. The task which processes this transaction scans the temporary directory in main memory to determine if the overlay in question is present on the fast disk. If not, the overlay is read from the slow disk, written to the fast disk, the temporary directory updated, and the overlay sent to the requesting application node. The fact that the transaction holds modification access to the temporary directory logical resource means that no other transaction can modify this directory while these operations are in progress, hence the temporary directory is not corrupted.

In the event that an entry for the requested overlay is present in the temporary directory, the GET OVERIAY transaction is not required to modify this directory. Hence it can reduce its access rights to the temporary directory logical resource to read-only in order to allow other transactions which require readonly access to this resource to proceed. However, since there is presently no mechanism in the common node operating system to allow such a reduction in access rights, the GET OVERLAY transaction simply retains modification access until it has been completely processed.

\section{Conclusions}

This extension to the main program and overlay manager subsystem should reduce the average time required for an application node to obtain a FORTRAN overlay from its present value of $\sim 480$ milliseconds to $\sim 340$ milliseconds. The corresponding reduction in the maximum time required to perform this operation should be from $\sim 550$ milliseconds to $\sim 400$ milliseconds. At present all subsystem code required for the extension has been written and is undergoing tests in the laboratory. It is hoped that a sufficient number of users will consider the extension valuable so that the exercise can be declared a success.

\section{Acknowledgements}

Almost everyone in the Systems Group at Brookhaven's Instrumentation Division has helped to implement the FORTRAN programing subsystem on the Reactor Experiment Control Facility. The program development node and control/manipulation node batchstreams were written by D. G. Dimmler and N. Greenlaw. The application node operating system was constructed by D. G. Dimmler, while M. A. Kelley contributed the assembly language subroutines to control the speclal purpose experiment electronics. The first version of the common node tasks belonging to the subsystem were written by D. G. Dimmler and completely rewritten in the process of adding the fast disk buffer facilities by $R$. D. Beck. Both the draft and final manuscript for this article were typed by B. D. Gaer. 
1. D. G. Dimler; Functional Distribution - An Architecture for Multi-User Computer Networks in Instrumentation. IEEE Trans. Nuc1. Sc1., NS-21, 838 (Feb. 1974).

2. D. G. Dimmler, N. Greenlaw; M. A. Kelley, D. W. Potter, S. Rankowitz, and F. W. Stubblefield; The Brookhaven Reactor Experiment Control Facility A Distributed Function Computer Network -. IEEE Trans. Nuc1. Sc1., NS-23, 398 (Feb. 1976).

3. Digital Equipment Corporation PDP-11/40.

4. PERTEC D3321-M012-NWU moving head disk.

5. Digital Equipment Corporation TC-II DECtape controller and TU56 dual DECtape drive.

6. Digital Equipment Corporation CR-11.

7. Versatek, Inc. D1100A line printer/plotter.

8. Digital Equipment Corporation DECwriter.

9. Disk Operating System (DOS), Version 9, September 1973, Digital Equipment Corporation, Maynard, Massachusetts.

10. Items 2-7 are software processors which execute under the operating system listed as Reference 9, above. They are, respectively, EDIT-11, FORTRN-11 (a compiler for FORTRAN IV),

MACRO-11,

LIBRARIAN-11,

LINK-1I, and

PIP-11.

11. FORTRAN Object Time System (OTS-11), Version 22, September 1973, Digital Equipment Corporation, Maynard, Massachusetts.

12. DECtape is a trademark of Digital Equipment Corporation.

13. Here, "global" means recognized by all logical program units within one main program-overlay set.

14. F. W. Stubblefield; Continuous Sharing of a Record-Oriented Output Device within a Distributed Function Laboratory Computer Network. IEEE Trans. Nuc1. Sci., NS-23, 423 (Feb. 1976).

15. F. W. Stubblefield; A File Management for Experiment Control Parameters within a Distributed Function Computer Network. IEEE Trans. Nucl. Sci., NS-24, 460 (Feb. 1977).

16. F. W. Stubblefield and D. G: Dimmler; Transaction Processing in the Common Node of a Distributed Function Laboratory Computer System. IEEE Trans. Nucl. Sc1. NS-22, 473 (Feb. 1975).

17. F. W. Stubblefield; Logical and Physical Resource Management in the Common Node of a Distributed Function Laboratory Computer Network. IEEE Trans. Nucl. Sci., NS-23, 406 (Feb. 1976).
18. F. W. Stubblefield and D. G. Dimmler; A Task Scheduler and Service Subsystem for the Common Node of a Distributed Function Laboratory Computer Network. IEEE Trans. Nucl. Sc1., NS-23, 413 (Feb. 1976).

19. F. W. Stubblefield; Conversion of an Operating System Oriented towards Transaction Processing from Two to Three Modes of Logical Address Space. To be presented at 1977 Nuclear Sclence Symposium.

20. Digital Development Corporation DMS-11. The controller is model number 20560; the disk drive is model number $6200-128$. 


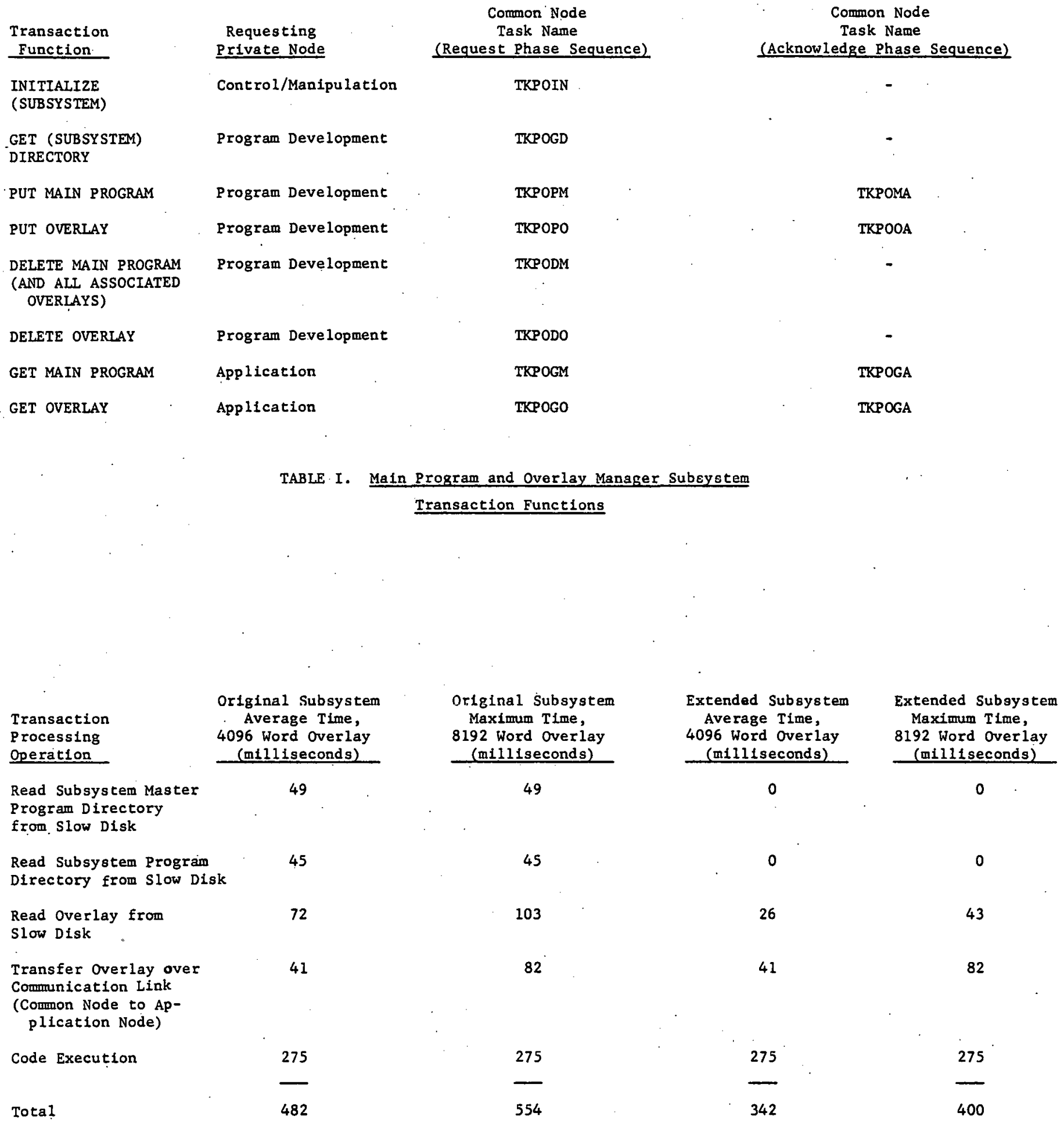

TABIE II. Transaction Processing Times for GET OVERIAY Function 


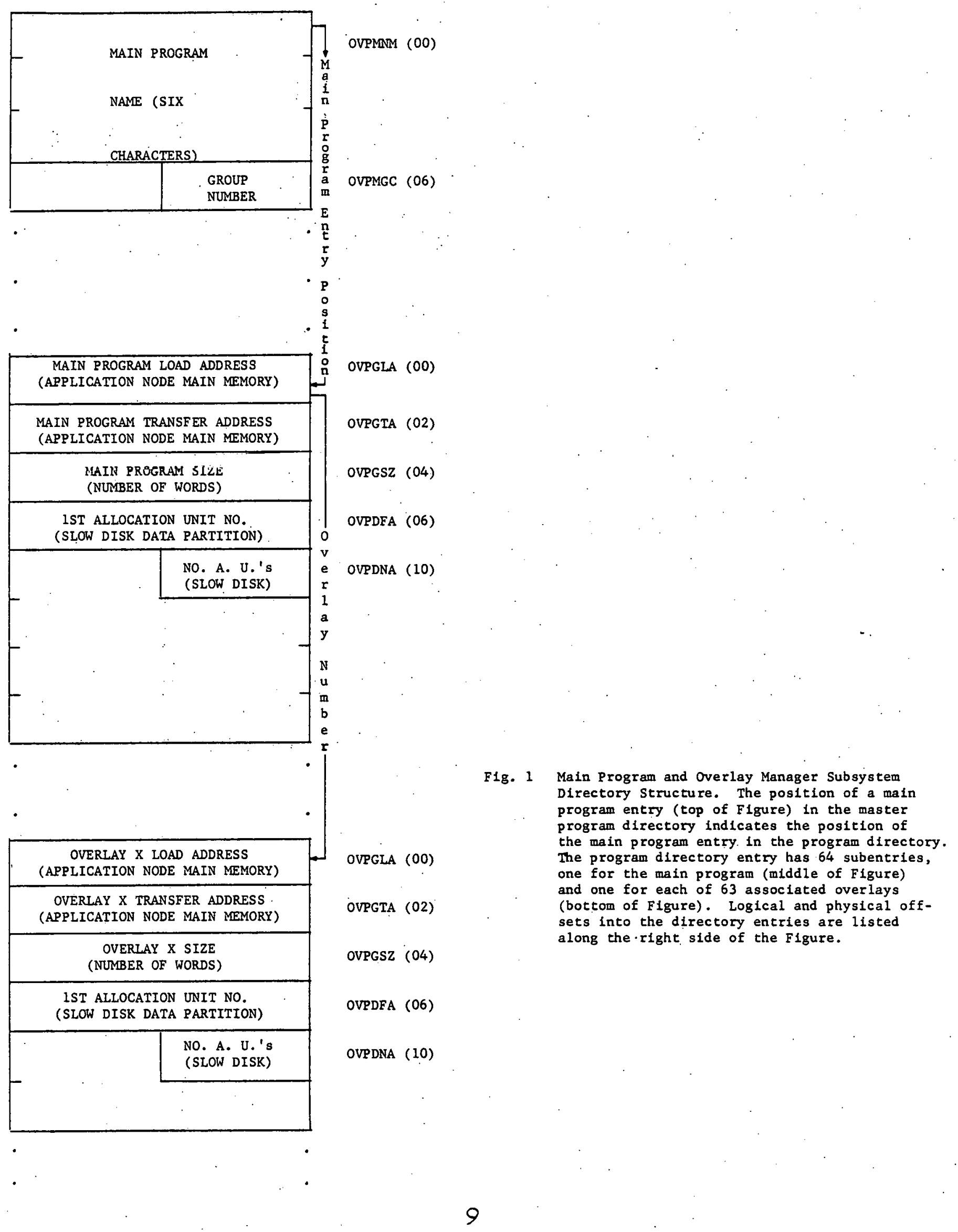

